\title{
Method for Clustering and Identification of Elements of Real Engineering Sites on The Basis of Dynamic Logic
}

\author{
Leonid Perlovsky* \\ Northeastern University, Psychology Department, USA \\ *Corresponding author: Leonid Perlovsky, Northeastern University, Psychology Department, USA
}

\begin{tabular}{l} 
ARTICLE INFO \\
\hline Received: 㓞 February 03, 2020 \\
Published: 靟 February 10, 2020
\end{tabular}

Citation: Leonid Perlovsky. Method for Clustering and Identification of Elements of Real Engineering Sites on The Basis of Dynamic Logic. Biomed J Sci \& Tech Res 25(3)-2020. BJSTR. MS.ID.004210.

Abbreviations: AI: Artificial Intelligence; NMF: Neural Modeling Fields; DL: Dynamic Logic; CC: Combinatorial Complexity; LIDAR: Light Identification Detection and Ranging

\begin{abstract}
Currently there is a contradiction between availability of various new equipment, which provides a stream of digital video data, in particular in the form of point clouds from mobile laser scanning, and the lack of adequate efficient methods of information extraction and analysis. This project is aimed at resolving this contradiction on the basis of neural modeling field theory and dynamic logic (DL) proposed by L. I. Perlovsky. The main result of the project will be a method of extracting information from digital video data in the form of hybrid clouds of mobile laser scanning data points for their analysis based on neural modeling field theory and DL. The success of this project depends on the successful integration of approaches from various fields of science and technology (interdisciplinarity): artificial intelligence, pattern and object recognition, logic, algorithm theory. The significance of the development of the proposed method is to create a fundamental theoretical basis for new application algorithms and software in the field of autonomous driving, "smart city" projects, ensuring safety for sites of various purposes, etc. The scientific novelty of the proposed method is that it will solve, by a fairly new method, the relevant problem of extracting and analyzing information from a not particularly traditional type of digital video data represented by a hybrid cloud of laser scanning points. This will allow to significantly expand the existing boundaries of knowledge in the field of extraction and analysis of information from various digital video data. The main hypothesis of the research is that the new method based on $\mathrm{L}$. I. Perlovsky's neural modeling field theory and DL will improve the performance of relevant calculations and close the existing gaps in the use of various digital video data.
\end{abstract}

\section{Short Communication}

Digital image and video processing are one of the most resource-intensive tasks and at the same time -important and relevant, closely related to the problem of artificial intelligence (AI) and modeling of cognition. Its frequent combinatorial complexity complicates the already difficult task: partition and recognize -partition video data (video image) into meaningful objects and recognize their essence, classify them. Mathematical logic offers to solve recognition problems by iterating over all known images and comparing them with the presented one. Even with a modestly sized set of options, the number of combinations to compare would be huge. At the same time, there are many separate methods and concepts in this field, often used together: decision trees, support vector method, convolutional neural networks, deep learning, K-means, latent Dirichlet allocation, etc.
L. I. Perlovsky has developed an original approach to cognition modeling based on the theory of neural modeling fields (NMF) and dynamic logic (DL) [1-3] that we propose for analyzing various digital video data. While ordinary logic operates with precise statements like "this is a chair", DL is a vague-to-crisp process. DL implements the causal principle of cognition, including object recognition: at each moment the state of the NMF is determined by the previous state. This approach, on one hand, is based on a detailed analysis of the problems of cognition modeling in AI (the shortcomings of formal logic; combinatorial complexity (CC)) and, on the other hand, on data from psychology, philosophy and cognitive science about the basic mechanisms of cognition. Perlovsky's analysis of problems in cognition modeling actually has a greater significance, and the overcoming of these problems 
can lead to new formalizations of the cognition process. Thus in [4] a generalization of the theory of NMF and DL is obtained in the form of phenomena and cognition DL. These logical systems are formulated in the most general terms: the relationship of generality, uncertainty, simplicity; the problem of maximizing similarity with empirical content; the learning method. Perlovsky L. I. notes that presently available neural networks are too simple to explain human cognition, and the NMF seek not only to improve methods for solving practical problems, but also to take a step towards explaining cognition.

The structure of NMF corresponds to our knowledge of the neural organization of the brain. The versatility of cognition and the wonder of the human brain are explained in part by dynamic logic. With standard Aristotelian (exact/"crisp") logic we encounter a lack of adaptability and a combinatorial explosion/complexity of computation (in particular, the "curse of dimensionality" as well). Logic, according to Aristotle, is a tool for expressing previously made decisions, not a mechanism of cognition, i.e. making new decisions. It is this multifaceted mechanism of NMF/DL and its underlying theory that we propose to be applied to various tasks related to the processing and extraction of information from various digital video data. The following is an overview of current research in the field of intelligent methods of extracting information from various digital video data with an analysis of the problems faced by researchers. At the same time, to even more clearly determine the relevance of the proposed method for the development of solutions to current problems, we present the prospects of using the method based on the NMF/DL to solve such problems.

Thus, [5] describes the different methods used in biometrics and lists the works with indication of used methods, features, and data sources. Among the open problems mentioned in [5] are statistical modeling and mathematical analysis of "soft" biometrics, including the processing of big data (for example, over 1.2 billion subjects in the Indian project UIDAI) and improving the accuracy and performance of such an algorithm. It is in fact the NMF/DL method that is capable of working quickly with big data without losing accuracy. In addition, it allows one to create statistical models. Work [6] addresses the problem of recognition of road signs and presents a table of works with indication of used methods, signs, and an assessment of recognition success, as well as the authors' own methods. This topic is an important part of one of the most important challenges of the 21st century: autonomous driving. The topic is also important for the inventory of signs on the country's roads and the tracking of their condition [6-8]. As with biometrics, there are certain obstacles to effective recognition: sign variability, lighting, shadow, orientation, etc. The authors of [6] work with a test base of 11000 signs. The NMF/DL method, should it have a dozen such bases, could help solve the problem of processing in an acceptable timeframe, and this would increase the accuracy along with performance.
Moreover, part of the task of autonomous driving is simultaneous detection of a sign in real time (and other objects: pedestrians, other cars, trees, traffic lights, etc.), its recognition, tracking, combining new information with information about all objects and information from all other sensors of the car, and finally, on the basis of all this, to give the car appropriate instructions. In addition to the large amount of data as such, the difficulty lies in the fact that all of this data must be linked together. The "associative" aspect of the NMF/DL method will solve this problem. A close cousin of autonomous driving is UAV (unmanned aerial vehicles) autonomous flight, for which it is also important to quickly recognize objects and landscape and make decisions. In addition to military applications, UAVs are also useful for scanning cities from a height to create their 3D models. In [7] the authors deal with this latter topic and write that LIDAR (Light Identification Detection and Ranging) is in demand because of the possibility of obtaining digital video data of high density and high image accuracy. At the same time, breakthroughs in the development of sensor technology, primarily laser, and of their corresponding algorithms allow us to efficiently and accurately build models based only on 2D images from cameras (including consumer-class) attached to the UAV.

The DL algorithm is able to solve both problems (LIDAR and 2D images from cameras). Then it would be possible to compare the accuracy of the two approaches, and, in the case of a clear victory for LIDAR, use LIDAR instead of cameras, as DL will allow to do so effectively, despite the high volume of data. In [7] the authors point to the instability of existing UAVs, which results in images being blurred and poorly structured. The "associative" aspect of the NMF/DL method will solve this problem as well. In addition to photo and video recording of road signs and entire cities, civil and military engineers also record roadways, buildings, factories, bridges and other infrastructure to monitor their condition and identify defects: cracks, breakdowns, vibrations, etc. Photo and video recording allow to more massively monitor the condition of objects, as well as to avoid risking lives of climbers who have to climb bridges, tall buildings, etc. Scientists have developed many approaches to the processing of such digital video data. A detailed review of the approaches is given in [8]: convolutional neural networks, deep learning, etc. Work [8] also describes the difficulties and challenges for scientists in this field, including the fact that a person still copes with the task of recognizing objects better than a computer program, thanks to knowledge of the situation context, correct identification of a structure's damaged part, knowledge of its importance in the design, etc. We propose linking all these aspects together using the NMF/DL method.

While [5] addresses biometric markers of the body such as the face, moles and tattoos, in [9] the authors cite their own method of processing the face in the context of medico-emotional well-being based on principal component analysis, a deep belief network and local direction-based robust features. Article [10] proposes a 
method, and article [11] an overview of the topic of recognizing abnormal crowd behavior. Such technology can help the police to recognize upcoming riots on city streets. Article [12] considers the recognition of human actions and suggests methods. This technology will be useful in airports and railway stations, where it is necessary to track individual actions of various individuals, and not the whole crowd. It will also find use in medicine and sports. The NMF/DL method has great prospects in improving the efficiency of solving such problems. The huge volume of unstructured video data from a plethora of cameras at railway stations and airports requires a radically new approach to their processing. This issue is touched upon in [13]. The NMF/DL method can potentially cope with this due to its performance. In [14] the issue of extraction of useful information from various data of past floods is discussed. In [15] the authors propose a way to significantly reduce the amount of data: to extract planes from it, since buildings and many objects often consist of them. In addition to planes, other features can be extracted; more on this in [16]. Work [17] develops a method for extracting text from video.

As stated in all aforementioned publications, any digital photo and video data typically contain different kinds of noise and clutter: leaves on the road, raindrops on a street sign, dust in the air, reflections, etc. In the NMF/DL method it is possible to specify a noise/clutter model which would take upon itself the corresponding noise points, such that the remainder of the points can be "cleanly" associated with the main models. Works [18-20] present the results of the development of methods and algorithms for processing laser scan and photography hybrid data. It is concluded that mobile laser scanning data is very promising from a practical point-of-view. At the same time, this type of laser scanning produces results in the form of a stream of inherently heterogeneous digital video data However, there are practical difficulties in finding solutions for this kind of real (rather than test) video data of huge size. This requires the development of fundamentally new methods of processing and analysis of this type of video data. Therefore, for the study of possible applications we chose a model of neural model fields, proposed and constructed by Perlovsky L.I., which allows to overcome the "curse of dimensionality" in recognition problems and interpreting a number of features of thought processes important for the analysis of video data $[1,21]$

\section{Methods and Materials}

Data from several sources were used. Outside scans of a European village were obtained from Semantic3D (bildstein station1_xyz_intensity_rgb). Inside scans of an office space were obtained from ISPRS's case study \#5. Our own scans of geometric blocks were used as well. Calculations were performed in MATLAB R2018a on a 64-bit Windows 10 Pro with 64 GB RAM, an 8-core Intel Xeon E5 CPU and an NVIDIA Quadro P6000 GPU. Most heavy-duty calculations were performed on the GPU. The algorithm was written in accordance to DL (dynamic logic) developed by L. I. Perlovsky
[1-3]. We used the clustering-specific version (also developed by Perlovsky) as opposed to the general one. The following Gaussian likelihood measure for conditional similarities was used:

$$
\ell(X(n) \mid m)=(1 / 2 \pi)^{d / 2} \operatorname{det} C_{m}^{-1 / 2} \bullet \exp \left[\frac{-\left(X(n)-M_{m}\right)^{T} C_{m}^{-1}\left(X(n)-M_{m}\right)}{2}\right]
$$

where $\mathrm{X}(n)$ is the input at data point $\mathrm{n}, \mathrm{Cm}$ are the covariance matrices for models (i.e. clusters) $\mathrm{m}=1, \ldots, \mathrm{M}$ and $\mathrm{Mm}$ is the model center expressed in the coordinates of $\mathrm{X}(n)$. The data point $\mathrm{X}(n)$ is generally in the form of a vector consisting of 3D spatial coordinates plus, optionally, intensity or color (RGB) or both. Model centers are initialized to randomly fall within the point cloud's boundary box and not too close to the boundary. Covariances are initialized random and large -on the order of the whole point cloud's size. Association variables, model rates (priors) and similarity measure, respectively, are given by

$$
\begin{aligned}
& \frac{f(m \mid n)=r_{m} \ell(n \mid m)}{\sum_{m^{\prime} \in M} r_{m} \ell(n \mid m)^{\prime}} \\
& r_{m}=(1 / n) \sum_{n \in N} f(m \mid n) \\
& L=\prod_{n \in N}\left[\sum_{m \in M} r_{m} \ell(n \mid m)\right]
\end{aligned}
$$

General DL equations for models and parameters are replaced by more clustering-specific equations (also given by Perlovsky):

$$
\begin{gathered}
M_{m}=\left(1 / N_{m}\right) \sum_{n \in N} f(m \mid n) X(n) \\
C_{m}=\left(1 / N_{m}\right) \sum_{n \in N} f(m \mid n)\left(X(n)-M_{m}\right)\left(X(n)-M_{m}\right)^{T}
\end{gathered}
$$

A mechanism has been developed for putting superfluous and redundant models to sleep and, on the other hand, waking up / creating new models when more are needed. So far it has not given entirely satisfactory results and is hence not used. In the future it may be useful to use it to automate the amount of models $M$ used instead of presetting the number upon initialization. Initially, the algorithm was used in one go, with the amount of models $M$ set at numbers like 25 or 60 . Later, a method giving much better results was found: if one wants, say, $M=25$ clusters, then initially the entire point cloud is broken down into 5 clusters, and then each of the original 5 clusters is broken down further into 5 clusters each, resulting in 25 clusters. In the end, it means each point in the cloud will have been clustered twice by two separate instances of the DL algorithm. This method (we call it " $5 \times 5$ ") not only gives much more sound results, but also ends up in faster calculation time. It also allows for much larger data sets and many more models $M$ to be assigned, since in the $1^{\text {st }}$ computation instance the computer has to handle $5 \mathrm{x}$ less models compared to the "in one go" method, and in the $2^{\text {nd }}$ instance, 
in addition to handling $5 \mathrm{x}$ less models, it also handles $5 \mathrm{x}$ less input data in each computational branch of the instance.

\section{Results and Discussion}

Our experiment focuses on clustering in just the 3 spatial coordinates because color and intensity from a laser scan can have too great a negative influence on the final clustering. A demonstration of this is below in (Figure 1). For the original shots we use a color scale for the intensity parameter (not for RGB color). The coloring in the resulting shot shows clustering (i.e. each color corresponds to its own cluster). As seen, intensity of a point depends a lot on angle of incidence, which is useful for discerning a façade from a roof, but is bad when a façade wall is round, or in the case of the car door, where the lower and upper halves, which are at slightly different angles, give off different intensities of the reflected laser beam. The clustering that results when intensity differences are carried over into the models is unsatisfactory. It also appears that reflected beam intensity depends on the material the beam hits, as seen on the car wheels: the rubber tire has one intensity, while the metal hub has a different intensity. This does not result in a meaningful clustering.
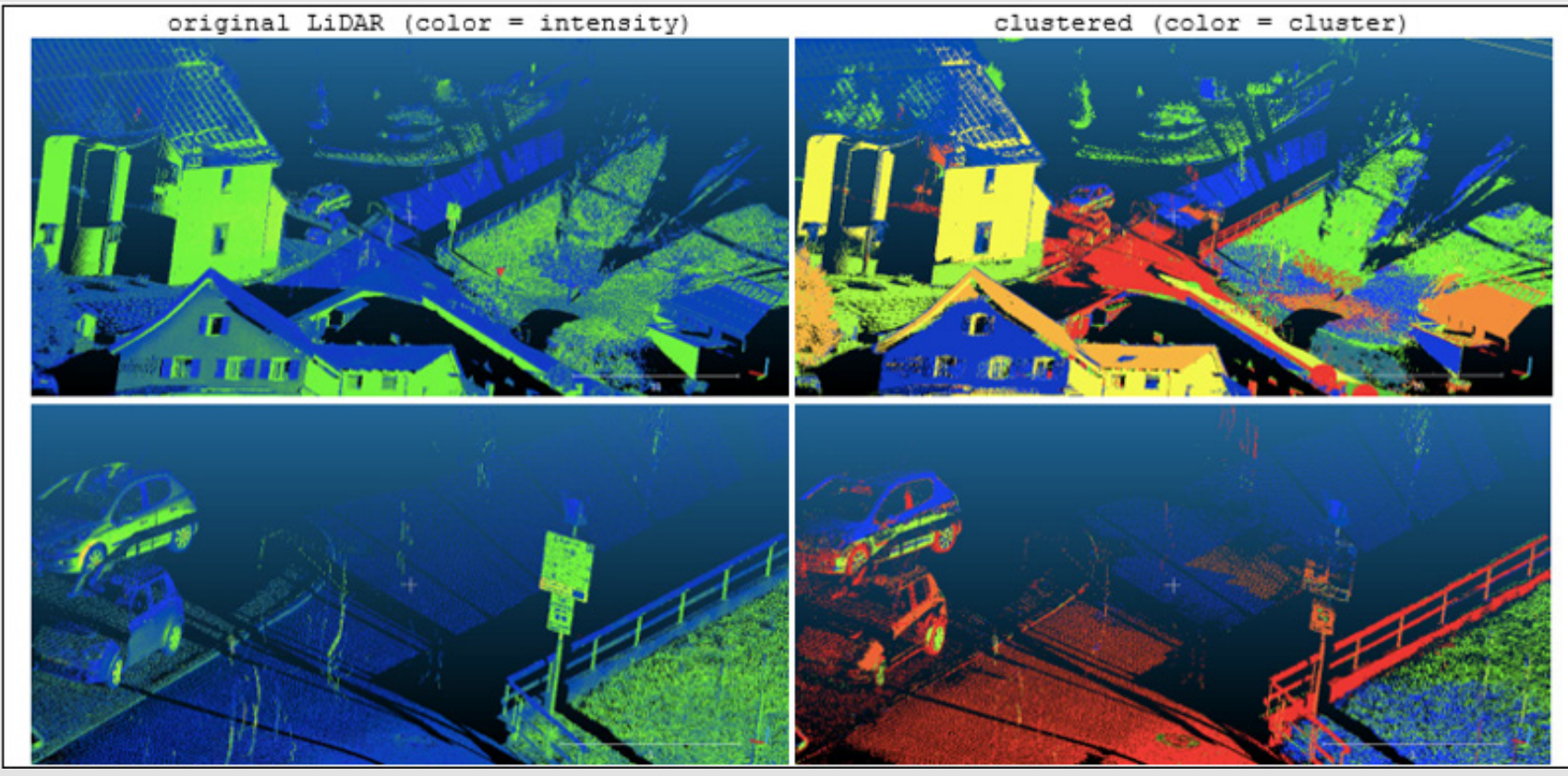

Figure 1.

Furthermore, we can see that where different intensities are mixed together chaotically, as they are on the field, the algorithm has given a similarly noisy intertwining of clusters. This may find use (in conjunction with the aforementioned dependency on the material of an object) in a crop field, where different types of crops must be differentiated from each other or from parasites or from the ground, but we have not performed such testing here, instead focusing on objects of infrastructure. It may make sense, however, to try such testing. Hence, as mentioned, we mostly continue applying our clustering algorithm to only the 3 spatial dimensions. The main objective is to break down an enormous point cloud into useful subsets. After that, it is entirely possible to run further instances of the algorithm on the obtained clustering result in more than just the 3 spatial dimensions, i.e. include color or intensity if it would seem to give a better result, or to altogether run a different type of algorithm on these subsets, such as one of object/pattern recognition.

In (Figure 2) below we demonstrate results of clustering the European village scan from Semantic3D into 25 clusters plus 1 noise cluster using the $5 \times 5$ method described in "Methods and materials", and compare it to the original " 25 clusters (plus 1 noise cluster) in 1 instance" method. Noise cluster is always colored light gray. Several successes and mishaps can be seen. The bottom screenshot, when compared to the 1 st one, shows that the $5 \times 5$ method generally results in a slightly sounder clustering, mainly manifested in objects not being broken up into random pieces as often. On the other hand, even in the final screenshot we still see parts of trees and buildings clustered together. Furthermore, we see one such cluster neighboring another such cluster containing parts of the same trees and buildings, and these 2 clusters are split by an approximately horizontal plane for no apparent reason. Granted, the algorithm is only one of clustering and not classification, hence it is not, in its bare form, smart enough to discern between objects.

It is important to mention the enormous effect that the positioning of the LiDAR scanner has on the clustering. In the final screenshot in (Figure 2) we see a green cluster in the middle - a roughly round patch of ground, no different than the ground around it. In the middle of it is where the LiDAR scanner was positioned. 
Hence, nearby it there is a much higher density of points than further away. The difference in density within the image has a large effect on the final result, since cluster weights depend on the relative number of points in them. This is why we see an accumulation of small-volume clusters near the scanner position, with largervolume clusters farther away from the scanner. To resolve this, a density readjustment subfunction could be implemented within the algorithm, or, alternatively, an initial down sampling (or a voxeling) could be performed in input data pre-processing before implementing DL. Also, if done correctly, working simultaneously on several scans from different positions could improve the result. Of the aforementioned, down sampling was tried, as was removing the ground, as well as a combination of these two approaches. Surprisingly, none of these methods gave better results, though down sampling helped reduce the concentration of small-volume, high-density clusters near the scanner position.

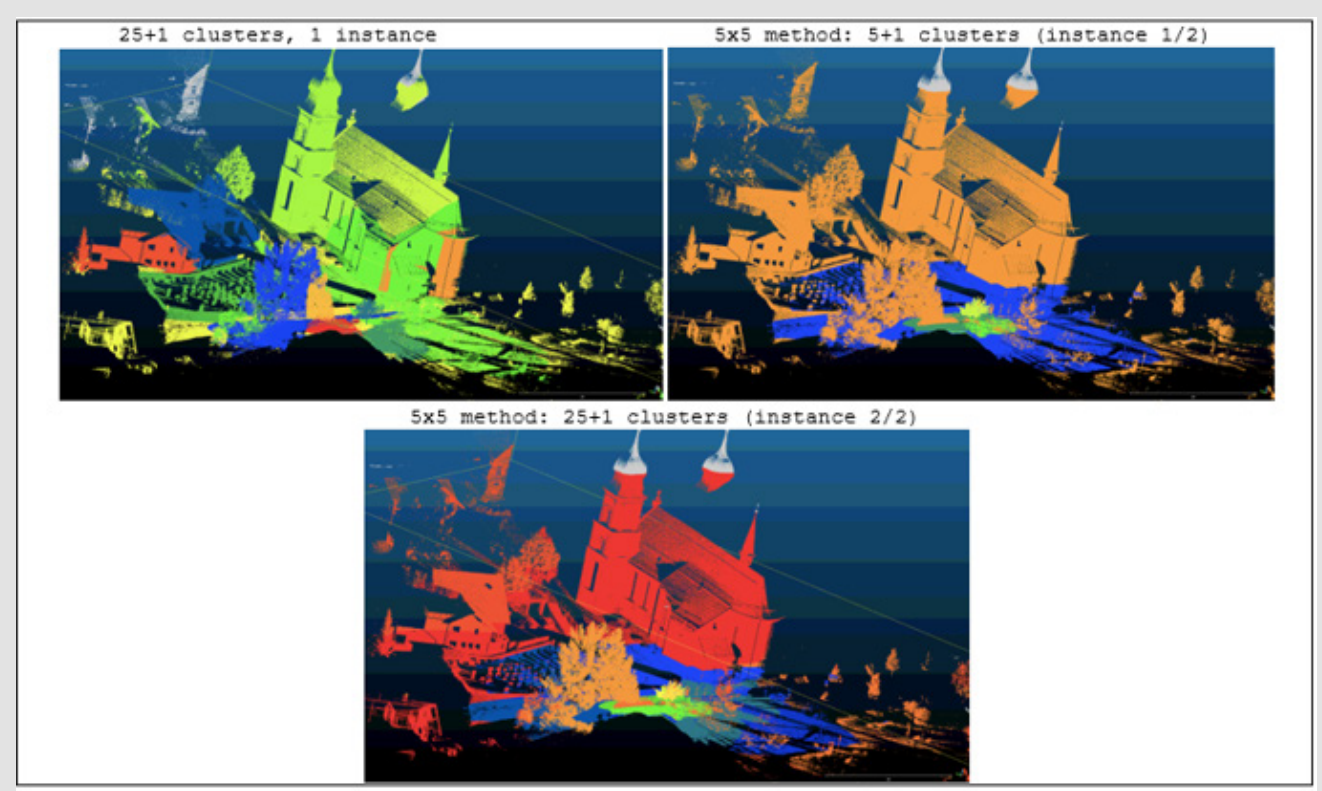

Figure 2.

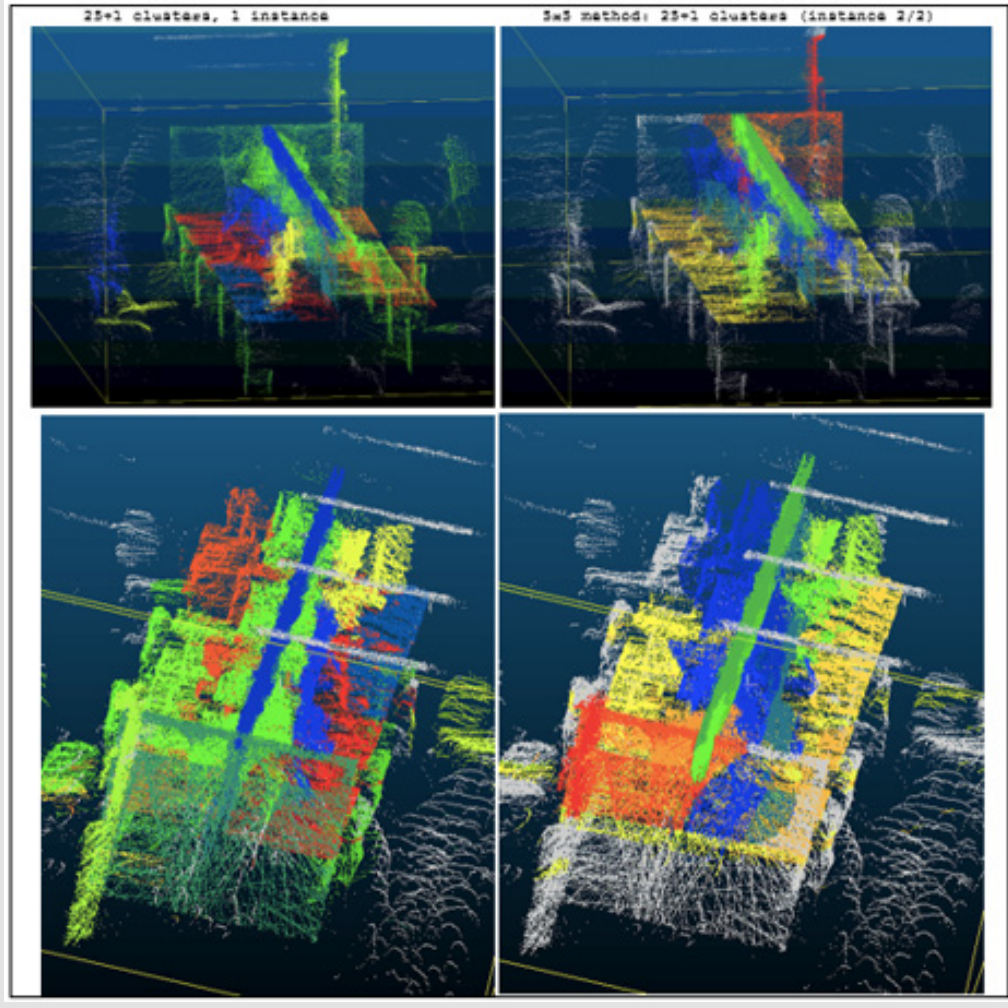

Figure 3. 
Also, since the algorithm involves gradient ascent, it is possible that the algorithm stops at a local maximum instead of a global one, though this is generally avoided by the vague-to-crisp aspect of the DL algorithm. Nonetheless, we tested this phenomenon by running the algorithm 50 times with randomly initiated cluster centers each time and picking the result with the greatest value of the likelihood function. This did not lead to a noticeably better result. Our DL algorithm was also tested on a subset (1 room) of ISPRS data (Case Study 5). The LiDAR data did not include an RGB reading -only intensity, which we ignored. Below in (Figure 3) we demonstrate the results from 2 different POVs and using the same 2 methods as before. As before, noise is colored gray. The most striking thing about these results is how well the tabletops and several other entities are separated from other objects, even if the tabletops themselves are split into several clusters. A clearer demonstration of this is below in (Figure 4) from different POVs. For clarity, the tabletops have been gathered into one cluster and recolored orange. The same is done with the top of the divider between tables (green). Bounding boxes are drawn for each of these 2 entities to show how flat/long they are. These 2 entities are displayed separately from everything else. The "everything else" is displayed in separate images from several POVs and maintains its original coloring (clutter points are excluded altogether).

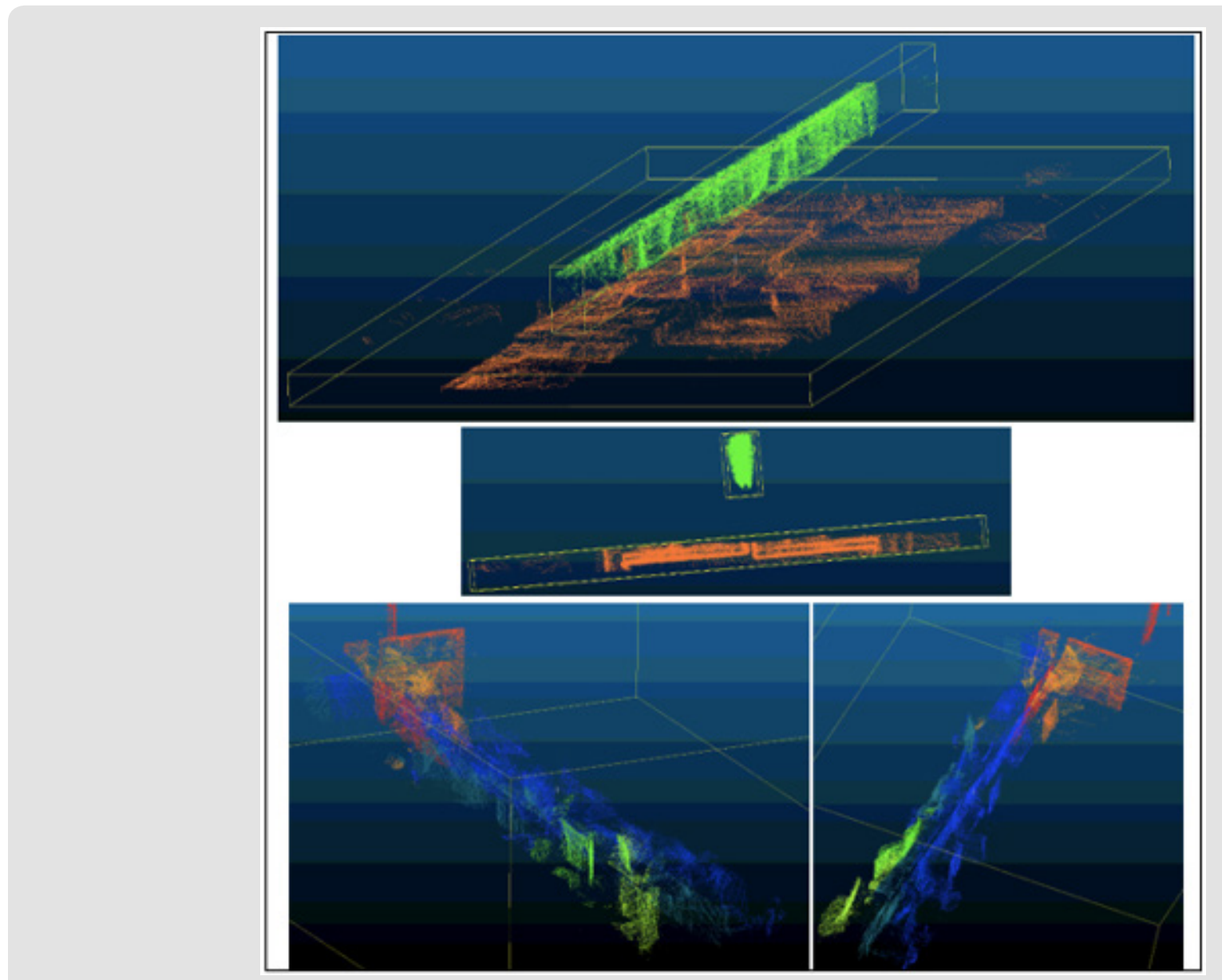

Figure 4 .

The "everything else" is clustered well in some ways, worse in others. The dark-orange vertical divider at the very end of the table is well-separated, though a large part of it is included in the lightorange cluster, which includes other things among it. The green/ yellow objects are actually several clusters that manage to fairly decently separate computer monitors. However, the blue and bluegrey clusters are a bit nonsensical. They go from one side of the long table divider to the other, i.e. are a bit skewed. Compared to the clustering of the outside scan (Bildstein), the indoor data seems to cluster better under our DL algorithm. Indeed, we see a better separation of objects and don't see as much chaotic splitting and overlapping, such as when half a tree would be clustered together with half a building, and the other halves of these objects would be in a $2^{\text {nd }}$ cluster. And though a tabletop, for example, may be split between 4 different clusters, it is trivial to join these clusters up into 1 due to the aforementioned separation of objects. A demonstration of this joining of clusters was already presented above.

Quick computation time (relative to other methods) is a big advantage of using DL. For example, with the Bildstein file, which contains about 29.7 million points, if we cluster over the 3 spatial dimensions, set the number of models (clusters) to $M=25$ plus 1 
noise model, then on our computer we achieve a computation time of just 34 seconds per 1 iteration of the main loop in the algorithm. Generally, in 10-20 iterations of this main loop a decent result can be achieved, but 40-50 are recommended. Similarly, if we use the $5 \times 5$ method with a limit of 40 iterations per branch/instance, i.e. we break down the cloud into 5 clusters (plus noise) in 40 iterations, then additionally break up each of the 5 resulting clusters into 5 more clusters each in 40 iterations, this takes 512 seconds on our computer, which, divided by 40 , gives us just 12.8 seconds, which is $2.7 \mathrm{x}$ faster than the 34 seconds in the 1 st method ( 1 instance, $M=25+1$ ). If we cut this time down in half by halving the number of iterations, then we encounter a loss of $4 \%$ in the shifted loglikelihood.

DL theory dictates that the computation time of DL is linear in the number of points, clusters and parameters, while in fact the results we achieved seemed to be better: DL is sublinear in all three. This may be attributable to the use of a GPU, as generally without a GPU (using standard RAM) the computation time was linear in the number of points, clusters and parameters. In fact, it is possible that calculation time with a GPU is sublinear up to a certain number of points/clusters/parameters, after which it approaches an asymptote, i.e. is linear, but this is not possible to test rigorously, since the GPU would have to be used in several full capacities.

\section{Conclusion}

This article describes the results of applying our DL-based clustering algorithm to LIDAR scans of a village street and inside an office space. The DL algorithm is able to work quickly with millions of points. All results presented here concern clustering in 3 spatial coordinates, i.e. ignoring the interfering color and intensity parameters, though these parameters can be useful in other cases, for example, in scans of agricultural fields. We use 2 clustering methods: in the first, segmentation is carried out simultaneously for 25 models (plus noise) in 1 instance (run) of the algorithm; the second (the so-called " $5 \times 5$ method") also clusters into 25 models (plus noise); however, it does so in 2 consecutive instances of the algorithm, where the $1^{\text {st }}$ run performs an initial segmentation into 5 clusters (plus noise), and in the 2 nd -each of the 5 initial nonnoise clusters is divided into a further 5 . The " $5 \times 5$ " method with the original Bildstein image allows clustering to be performed 2.7 times faster (29.7 million points in about 8 minutes), and the result is not worse than the one obtained by the 1 st (basic) method. Reducing the number of iterations (and therefore the calculation time) by half reduces the shifted log-likelihood by $4 \%$.

The best results are obtained when clustering the office space scan (ISPRS). The high-quality segmentation of the countertop and dividers stands out particularly. In the case of the village scan, we see satisfactory results. DL-clustering divides the space both into clusters that make sense and those that make less sense. To improve the result, we tried down sampling, removing the ground, and a combination of these two approaches. However, no improvement is thence shown. We also consider the possibility that the algorithm reaches a non-global local maximum in its gradient ascent; however, these doubts are refuted with high certainty: we ran the algorithm 50 times with arbitrary initializations, and all results gave approximately the same final likelihood.

\section{References}

1. Perlovsky LI (2006) Toward physics of the mind: Neural modeling field theory. Neuroinformatic 1(2): 175-196.

2. Perlovsky LI (2006) Toward physics of the mind: Concepts emotions consciousness and symbols. Physics of Life Reviews 3(1): 23-55.

3. Perlovsky LI (2007) Neural networks fuzzy models and dynamic logic. Studies in Fuzziness and Soft Computing 209(3): 363-386.

4. Kovalerchuk B, Perlovsky L (2008) Dynamic logic of phenomena and cognition. IEEE pp. 3530-3537.

5. Dantcheva A, Elia P, Ross A (2015) What else does your biometric data reveal? A survey on soft biometrics. IEEE Transactions on Information Forensics and Security 11(3): 441-467.

6. Balali V, Golparvar Fard M (2016) Evaluation of multiclass traffic sign detection and classification methods for US roadway asset inventory management. Journal of Computing in Civil Engineering 30(2): 04015022.

7. Rothermel M (2014) Fast and robust generation of semantic urban terrain models from UAV video streams. $201422^{\text {nd }}$ International Conference on Pattern Recognition pp. 592-597.

8. Spencer Jr BF, Hoskere V, Narazaki Y (2019) Advances in Computer Vision-Based Civil Infrastructure Inspection and Monitoring. Engineering 5: 199-222.

9. Uddin Z, Hassan M, Almogren A, Alamri A, Alrubaian M, et al. (2017) Facial expression recognition utilizing local direction based robust features and deep belief network. IEEE Access 5: 4525-4536.

10. Wang B, Ye M, Li X, Zhao F (2011) Abnormal crowd behavior detection using size adapted spatio temporal features. International Journal of Control Automation and Systems 9: 905.

11. Sreenu G, Durai MAS (2019) Intelligent video surveillance: a review through deep learning techniques for crowd analysis. Journal of Big Data 6: 1 .

12. Chou MK, Prasad M, Wu D, Sharma N, Li DL, et al. (2018) Robust feature based automated multi view human action recognition system. IEEE Access 6: 15283-15296

13. Gharbi H, Bahroun S, Zagrouba E (2018) Key frame extraction for video summarization using local description and repeatability graph clustering. Signal Image and Video Processing 13(3): 507-515.

14. Macchione F, Costabile P, Costanzo C, De Lorenzo G (2019) Extracting quantitative data from non-conventional information for the hydraulic reconstruction of past urban flood events. A case-study. Journal of Hydrology 576: 443-465.

15. Zhang G, Vela P, Karasev P, Brilakis I (2015) A sparsity-inducing optimization-based algorithm for planar patches extraction from noisy point cloud data. Computer-Aided Civil and Infrastructure Engineering 30(2): 85-102.

16. Jimenez M (2018) Summarization of video from Feature Extraction Method using Image Processing and Artificial Intelligence.

17. Wong EK, Chen M (2003) A new robust algorithm for video text extraction. Pattern Recognition 36(6): 1397-1406.

18. Badenko V, Tammsaar S, Beliaevskii K, Fedotov A, Vinogradov K, et al. (2019) Multithreading in Laser Scanning Data Processing. Lecture Notes in Computer Science 11619: 289-305. 
19. Badenko V, Fedotov A, Vinogradov K (2018) Algorithms of laser scanner data processing for ground surface reconstruction. Lecture Notes in Computer Science 10961: 397-411.

20. Fateeva E, Badenko V, Fedotov A, Kochetkov I (2018) System analysis of the quality of meshes in HBIM. MATEC Web of Conferences 170: 03033.

\section{ISSN: 2574-1241}

DOI: 10.26717/BJSTR.2020.25.004210

Leonid Perlovsky. Biomed J Sci \& Tech Res

(C) (P) This work is licensed under Creative

Submission Link: https://biomedres.us/submit-manuscript.php
21. Perlovsky LI (2017) Physics of the mind dynamic logic and monotone Boolean functions. Studies in Computational Intelligence 683: 193-231.

$\begin{array}{ll}\text { BIOMEDICAL } & \text { Assets of Publishing with us } \\ \text { RESEARCHES } & \text { - Global archiving of articles } \\ & \text { - Immediate, unrestricted online access } \\ & \text { - Rigorous Peer Review Process } \\ & \text { - Authors Retain Copyrights } \\ \end{array}$

